O Mediano

A Barceló

M de la Peña

D Gozal

A Agusti

F Barbé

\section{Hipersonolência diurna e variáveis polissonográficas em doentes com síndroma de apneia do sono}

\author{
Daytime sleepiness and polysomnographic variables \\ in sleep apnoea patients
}

\section{Resumo}

A síndroma de apneia obstrutiva do sono (SAOS) caracteriza-se por episódios repetidos de obstrução da via aérea superior, hipoxemia nocturna e fragmentação do sono. A hipersonolência diurna constitui um sintoma frequente nos doentes com esta síndroma, embora possa estar ausente. A sua presença representa uma limitação significativa da qualidade de vida do indivíduo, bem como um aumento da morbilidade e mortalidade da SAOS, visto ser um importante factor de risco para acidentes de viação e laborais, podendo originar alterações cognitivo-comportamentais relevantes.

O mecanismo desencadeante de hipersonolência diurna não é claro. Alguns autores relacionaram esta com a arquitectura anormal do sono, o que, no entanto, não foi confirmado por outros investigadores. Alguns estudos salientaram a relação entre a hipersonolência diurna e os níveis de dessaturação nocturna da oxi-hemoglobina, mas este facto não foi comprovado cientificamente.

O objectivo do presente trabalho foi analisar quais os mecanismos responsáveis pela sonolência diurna nos doentes com SAOS. Assim, numerosas variáveis registadas no estudo polissonográfico foram compara- das em dois grupos de indivíduos com esta patologia, apresentando um deles hipersonolência diurna inequívoca e o outro a total ausência da mesma.

Foram avaliados 65 indivíduos seguidos no Departamento Respiratório do Hospital Universitário do Son Dureta (Palma de Maiorca) que possuíam no registo poligráfico nocturno um índice de apneia/hipopneia $(\mathrm{IAH})>20 / \mathrm{h}$. A sonolência excessiva foi determinada utilizando a escala de sono de Epworth (ESE) e o teste de latência múltipla. A sua presença foi considerada sempre que o score de ESE $>10$ e o score do teste de latência múltipla $<5$ min. A ausência da mesma era determinada por um score de ESE $<10$ e um teste de latência $>10$ min.

Cerca de 25 indivíduos foram excluídos devido a discrepância entre os resultados das referidas determinações. Dos 40 doentes restantes, 23 apresentavam hipersonolência diurna e 17 não. Nenhum dos indivíduos sofria de doença crónica, nomeadamente DPOC, cirrose hepática, disfunção tiroideia, artrite reumatóide, insuficiência renal crónica ou fazia qualquer tipo de medicação.

O diagnóstico de SAOS foi estabelecido por polissonografia nocturna com registo de múltiplas variáveis, como

Eur Resp J 2007; 30: 110-113 
sejam electroencefalograma (EEG), electromiograma (EMG) mentoniano, electrooculograma, fluxo oronasal, movimentos toracoabdominais e oximetria de fluxo. Apneia foi definida como a interrupção de fluxo oronasal $>10$ s e hipopneia como uma redução $>50 \%$ do fluxo por um período $>10$ s associado a uma descida $>4 \%$ da $\mathrm{SaO}_{2}$ ou despertar. $\mathrm{O}$ índice de apneia/hipopneia (IAH) consiste no somatório do número de apneias e hipopneias por hora de sono. Os estádios do sono foram classificados de acordo com os critérios de Rechtschoffen e Kales. A latência do sono foi definida como o período compreendido entre o acto de deitar e os primeiros 30s do estádio I e a eficiência do sono como a duração do sono nocturno expressa como percentagem do tempo total de permanência no leito. De acordo com os critérios da American Sleep Disorders Association Task Force, os despertares foram definidos como despertares de origem respiratória quando ocorriam 3s após uma apneia, hipopneia ou ressonar, sabendo que existem outros tipos de despertares (espontâneos, relacionados com os movimentos periódicos dos membros e técnicos). O total de despertares foi determinado pela soma de cada tipo dos mesmos.

A hipersonolência subjectiva foi avaliada utilizando a ESE constituída por 8 itens a que são atribuídos um score de 0 a 3. A avaliação objectiva é obtida através do teste de latência múltipla de acordo com as guidelines internacionais. Um score $<5$ min traduz hipersonolência diurna e $>10$ min a sua ausência.

Foram estudados, no total, 23 indivíduos do sexo masculino com hipersonolência diurna (ESE: 17士3; teste latência múltipla: $4 \pm 1 \mathrm{~min})$ e 17 sem sonolência excessiva (ESE: $5 \pm 2$ e teste latência: $16 \pm 3 \mathrm{~min})$. Ambos os grupos presentaram um IAH similar $(62 \pm 18 / \mathrm{h}$ versus $60 \pm 20 / \mathrm{h}$ ). Os doentes com hipersonolência possuíam um tempo de latência inferior $(11 \pm 16$ versus

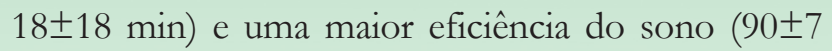
versus $82 \pm 13 \%$ ) do que os indivíduos sem sonolência excessiva. Os primeiros apresentavam uma menor oxi-

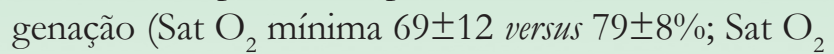
média $87 \pm 6$ versus $90 \pm 5 \%$ ).

A distribuição dos estádios do sono e o índice de despertar não diferiu entre os grupos.

Verificou-se, assim, que doentes com SAOS e sonolência diurna excessiva se caracterizavam por um tempo de latência do sono inferior, uma maior eficiência do sono e uma oxigenação nocturna pior em relação aos indivíduos sem hipersonolência diurna. A hipoxemia nocturna parece ser o factor determinante major da hipersonolência diurna dos doentes com SAOS.

\section{Comentário}

Com o intuito de estudar os factores determinantes da hipersonolência diurna em doentes com SAOS, foram comparadas numerosas variáveis do registo poligráfico do sono de dois grupos de indivíduos com a referida patologia que apresentavam ou não sonolência excessiva, sendo os parâmetros como idade, sexo e IMC sobreponíveis. O achado mais relevante do presente estudo foi a existência de um menor tempo de latência, maior efi- ciência do mesmo e pior oxigenação nocturna nos indivíduos com hipersonolência diurna. Não havia, no entanto, diferenças significativas em relação ao IAH, índice de despertar e a arquitectura do sono.

Verificou-se, assim, que doentes com hipersonolência diurna têm um sono mais eficiente, um menor tempo de latência e dormem mais tempo. Os autores acreditam que estas diferenças são, provavelmente, uma consequência e não uma causa da hipersonolên- 
cia. Estes factores parecem indicar que estes doentes se mantêm sonolentos ao longo de 24 horas.

Os indivíduos com sonolência em excesso apresentam apneias mais prolongadas, sugerindo, assim, existir um atraso nos despertares. Sendo o grau de gravidade do distúrbio respiratório sobreponível, podemos concluir que o IAH não nos esclarece sobre os mecanismos desencadeantes do sintoma referido. Alternativamente, observou-se que uma maior hipoxemia nocturna se correlacionava com a hipersonolência diurna, tendo-se verificado que os indivíduos com maior dessaturação de oxigénio nocturna apresentavam uma sonolência mais acentuada apesar de o IAH, índice de despertar e arquitectura do sono, serem idênticos em ambos os grupos. Este facto salienta o importante papel da dessaturação nocturna na patogénese da hipersonolência, como foi sugerido.

Zahn e colaboradores revelaram que a hipoxemia intermitente em regiões cerebrais de ratos, através de eventos oxidativos e inflamatórios, conduziam à perda de células neuronais e à manifestação de sonolência nos mesmos...

Conclui-se, assim, que a hipoxemia nocturna tem um papel major na patogénese da hipersonolência diurna dos doentes com SAOS.

\section{Mensagem}

- Doentes com SAOS e hipersonolência diurna apresentam um tempo de latência do sono inferior, uma maior eficiência do sono e uma pior oxigenação nocturna do que os indivíduos com SAOS e ausência de sonolência excessiva.

- A hipoxemia nocturna pode ser o factor determinante major da hipersonolência diurna na SAOS

\section{Bibliografia}

1. Bárbara C. Fisiopatologia da Síndrome de Apneia Obstrutiva do Sono (SAOS) - in MJ Marques Gomes e R Sotto-Mayor (Eds.) Tratado de Pneumologia. Lisboa 2003; vol I, secção K, cap. 77, pp. 1036-9.

2. Bárbara C, Pinto P. Síndrome de Apneia Obstrutiva do Sono. Diagnóstico e terapêutica. Monografia 2005.

3. Caeiro F. Aspectos actuais da Síndrome de Apneia do Sono. In Pneumologia Actual. 40. ${ }^{\circ}$ Curso de Pneumologia para Pós-Graduados. Clínica Universitária de Pneumologia FML/HSM. Lisboa 2007, pp. 103-5.

4. Johns MW. A new method for measuring daytime sleepiness: the Epworth Sleepiness Scale. Sleep 1991; 14:540-5. 5. Zhan G, Fenik P, Prateco D, Veasey SC. Inducible nitric oxide synthase in long-term intermittent hypoxia: hypersonnolence and brain injury. Am J Respir Crit Care Med 2005; 171:1414-20.

Fátima Caeiro 07.09.04 